\title{
A Cost-Effective Agent for Clinical Trial Assignment
}

\author{
Princeton K. Kokku, Lawrence O. Hall, Dmitry B. Goldgof, Eugene Fink, and Jeffrey P. Krischer \\ kokku@csee.usf.edu, hall@csee.usf.edu, goldgof@csee.usf.edu, \\ eugene@csee.usf.edu,jpkrischer@moffitt.usf.edu
}

Computer Science and Engineering, University of South Florida, Tampa, Florida 33620

\begin{abstract}
The purpose of a clinical trial is to evaluate a new treatment procedure. When medical researchers conduct a trial, they recruit participants with appropriate medical histories. To select participants, the researchers analyze medical records of the available patients, which has traditionally been a manual procedure. We describe an intelligent agent that helps to select patients for clinical trials. If the available data are insufficient for choosing patients, the agent suggests additional medical tests and finds an ordering of the tests that reduces their total cost.

Keywords - Medical expert systems, automated diagnosis, clinical trials.
\end{abstract}

\section{INTRODUCTION}

A clinical trial is an experiment with a new treatment procedure. When medical researchers test a new treatment, they recruit patients with appropriate health problems and medical histories. The selection of patients has traditionally been a manual procedure, and recent studies have shown that clinicians can miss up to $60 \%$ of the eligible patients [9, 10, 14, 26, 35, 38].

If the available records do not provide enough data, clinicians perform medical tests as part of the selection process. The costs of most tests have declined over the last decade, but the number of tests has significantly increased $[33,36]$, which is partially due to inappropriate ordering of tests $[1,25]$. Clinicians can reduce the cost by first requiring inexpensive tests and then using their results to avoid some expensive tests; however, finding the right ordering may be a complex problem.

The purpose of the described work is to automate the selection of patients for clinical trials and minimize the cost of related tests. We have developed an agent that identifies appropriate trials for each patient, and built a knowledge base for breast-cancer trials.

\section{Previous Work}

Researchers began to work on medical expert systems in the early seventies. Shortliffe et al. developed the MYCIN system, which diagnosed bacterial diseases $[5,30$, 31]. Its knowledge base consisted of if-then rules, which allowed for the analysis of symptoms and evaluation of the certainty of the diagnosis. Experiments showed that MYCIN correctly diagnosed common diseases, which led to the development of other medical systems [5, 19], such as NEOMYCIN, PUFF, CENTAUR, and VM. Shortliffe et al. created a system for selecting chemotherapy treatments, called ONCOCIN [32], which also evolved from MYCIN.

Lucas et al. constructed a rule-based system for diagnosing liver and biliary-tract diseases [16], but it often gave an incorrect diagnosis [12, 23]. Korver and Lucas converted the initial system into a Bayesian network, which improved its performance [13, 15].

Musen et al. built a rule-based system, called EON, that selected AIDS patents for clinical trials [20]. OhnoMachado et al. developed the AIDS $^{2}$ system, which also assigned AIDS patients to clinical trials [21]. They integrated logical rules with Bayesian networks, which helped to make decisions in the absence of some data.

Bouaud et al. created a cancer expert system, called ONCODOC, that suggested alternative clinical trials for each patient and allowed a physician to choose among them $[3,4]$. Séroussi et al. used ONCODOC to select participants for clinical trials at two hospitals, which helped to increase the number of selected patients by a factor of three [27, 28, 29].

Hammond and Sergot created the OaSiS architecture [11], which combined the techniques from earlier systems, including EON and ONCOCIN. Smith et al. built a system that assisted a clinician in selecting medical tests and reducing their number and cost [17, 18, 33].

Fallowfield et al. studied how physicians selected cancer patients for clinical trials, and compared manual and automatic selection [8]. They showed that expert systems could improve the selection accuracy; however, their study also revealed that physicians were reluctant to use these systems. Carlson et al. conducted similar studies with AIDS trials, and also concluded that expert systems could lead to a more accurate selection [6].

Theocharous developed a Bayesian system that selected clinical trials for cancer patients [24, 34]. It learned conditional probabilities of medical-test outcomes and evaluated the probability of a patient's eligibility for each trial. On the negative side, the available medical records were often insufficient for learning accurate probabilities. Furthermore, when adding a new clinical trial, the user had to change the structure of the underlying Bayesian network.

To address these problems, Bhanja et al. built a rulebased system for the same task [2]. We have continued that work, extended the system, and added a mechanism for reducing costs involved in patient selection.

\section{EXAMPLE}

We have developed an intelligent agent that helps to select clinical trials for eligible patients. It prompts a clinician to enter the results of medical tests, and identifies appropriate trials. If the available records do not provide enough data, the agent suggests additional tests. In Figure 1(a), we give a simplified example of eligibil- 


\section{(a) Eligibility criteria}

1. The patient is female.

2. She is at most forty-five years old.

3. Her cancer stage is II or III.

4. Her cancer is not invasive.

5. At most three lymph nodes have tumor cells.

6. Either

- the patient has no cardiac arrhythmias, or

- all tumors are smaller than 2.5 centimeters.

(b) Tests and questions

General information

What is the patient's sex?

What is the patient's age?

Mammogram, Cost is $\$ 150$

What is the cancer stage?

Does the patient have invasive cancer?

Biopsy, Cost is $\$ 300$

What is the cancer stage?

How many lymph nodes have tumor cells?

What is the greatest tumor size?

Electrocardiogram, Cost is $\$ 200$

Does the patient have cardiac arrhythmias?

Fig. 1. Example of eligibility criteria, tests, and questions.

\begin{tabular}{l|l}
\hline (a) Acceptance & (b) Rejection \\
sex $=$ FEMALE and & sex $=$ MALE or \\
age $\leq 45$ and $>45$ or \\
stage $\in\{\mathrm{II}, \mathrm{III}\}$ and & cancer $\in$ II, IV $\}$ or \\
invasive $=\mathrm{NO}$ and & invasive $=\mathrm{YES}$ or \\
lymph-nodes $\leq 3$ and & lymph-nodes $>3$ or \\
$\begin{array}{c}\text { (arrhythmias }=\mathrm{NO} \text { or } \\
\text { tumor-size } \leq 2.5)\end{array}$ & $\begin{array}{c}\text { arrhythmias }=\mathrm{YES} \text { and } \\
\text { tumor-size }>2.5)\end{array}$ \\
\hline
\end{tabular}

Fig. 2. Logical expressions for the criteria in Figure 1(a).

ity criteria for a clinical trial. This trial is for young and middle-aged women with a noninvasive cancer at stage II or III. When testing a patient's eligibility, a clinician has to order three medical tests (Figure 1b).

The agent first prompts a clinician to enter the patient's sex and age. If the patient satisfies the corresponding conditions, the agent asks for the mammogram results and verifies Conditions 3 and 4 ; then, it requests the biopsy and electrocardiogram data. If the patient's records already include some test results, the clinician can answer the corresponding questions while entering the personal data, before the agent selects test procedures. For example, if the records indicate that the cancer stage is IV, the clinician can enter the stage along with sex and age, and then the agent immediately determines that the patient is ineligible for this trial.

\section{KNOWledge BAse}

The agent's knowledge base includes questions, medical tests, and logical expressions that represent eligibility criteria for each trial. We give a simplified example of tests and questions in Figure 1(b), and logical expressions in Figure 2.

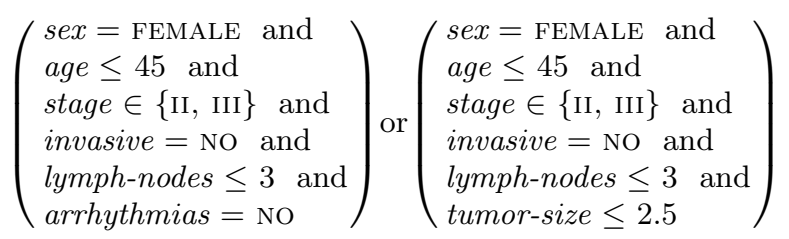

Fig. 3. Disjunctive normal form of the acceptance expression.

The agent supports three types of questions; the first type takes a yes/no response, the second is multiple choice, and the third requires a numeric answer. For example, the cancer stage is a multiple-choice question, and the tumor size is a numeric question. The description of a medical test includes the test name, dollar cost, and list of questions that can be answered based on the test results (Figure 1).

We encode the eligibility for a clinical trial by a logical expression that does not have negations, called the acceptance expression. It includes variables that represent medical data, as well as equalities, inequalities, "set-element" relations, conjunctions, and disjunctions (Figure 2a). In addition, the agent uses the logical complement of the eligibility criteria, called the rejection expression, which also does not have negations (Figure $2 \mathrm{~b}$ ). It describes the conditions that make a patient ineligible for the trial.

The agent collects data until it can determine which of the two expressions is TRUE. For instance, if a patient's sex is MALE, then the rejection expression in Figure 2(b) is TRUE, and the agent immediately determines that this trial is inappropriate. If the sex is FEMALE, the agent asks more questions.

If the knowledge base includes multiple clinical trials, the agent checks a patient's eligibility for each of them. It first asks for the tests related to multiple trials, and then requests additional tests for specific trials. After getting each new answer, the agent re-evaluates the patient's eligibility for each trial.

\section{ORDER OF TESTS}

If a patient's records do not include enough data, the agent asks for additional tests; for example, if the records do not provide data for the eligibility criteria in Figure 1, the agent asks for the mammogram, biopsy, and electrocardiogram. The total cost of tests may depend on their order; for instance, if we begin with the mammogram, and it shows that the cancer stage is IV, then we can immediately reject the trial in Figure 1 and avoid the more expensive tests.

We have explored heuristics for ordering the tests, based on the test costs and the structure of acceptance and rejection expressions. The heuristics use a disjunctive normal form of these expressions; that is, each expression must be a disjunction of conjunctions. For example, the rejection expression in Figure 2(b) is in disjunctive normal form, whereas the acceptance expression in Figure 2(a) is not. If the system uses ordering heuristics, it converts this acceptance expression into the disjunctive normal form shown in Figure 3. 
The agent chooses the order of tests that reduces their expected cost. After getting the results of the first test, it re-evaluates the need for the other tests and revises their ordering. The choice of the first test is based on three criteria. The agent scores all required tests according to these criteria, computes a linear combination of the three scores for every test, and chooses the test with the highest score.

1. Cost of the test. The agent prefers cheaper tests. For instance, it may start with the mammogram, which is cheaper than the other two tests in Figure 1.

2. Number of clinical trials that require the test. When the agent checks a patient's eligibility for several trials, it prefers tests that provide data for the largest number of trials. For example, if the electrocardiogram gives data for two different trials, the agent may prefer it to the mammogram despite its higher cost.

3. Number of clauses that include the test results. The agent prefers the tests that provide data for the largest number of clauses in the acceptance and rejection expressions. For example, the mammogram data affect both clauses of the acceptance expression in Figure 3 and two clauses of the rejection expression in Figure $1(\mathrm{~b})$. On the other hand, the electrocardiogram affects only one clause of the acceptance expression and one clause of the rejection expression; thus, the agent should order it after the mammogram.

\section{USER INTERFACE}

The agent includes a web-based interface that allows clinicians to enter patients' data through remote computers; the interface consists of five screens (Figure 4).

The start screen is for adding and retrieving patients (Figure 5). After a user enters a patient's name, the agent displays a list of the available trials (Figure 6). The user can choose a subset of these trials, and then the agent checks eligibility only for the selected trials. The next screen is for basic personal and medical data, such as sex, age, and cancer stage (Figure 7).

After the agent gets the basic data, it prompts the user for medical information related to specific trials (Figure 8). When the user enters medical data, the agent continuously re-evaluates the patient's eligibility and shows the decision for each trial. If the patient is ineligible for some trials, the user can find out the reasons by clicking the "Why" button. The interface also includes a screen for the review and modification of the previous answers, similar to the screen in Figure 8.

\section{EXPERIMENTS}

We have built a knowledge base for the breast-cancer clinical trials at the H. Lee Moffitt Cancer Center, applied the agent to retrospective data from 187 past patients and 57 current patients, and compared the results with manual selection by clinicians at the cancer center.

We summarize the results for the past patients in Table I, and the results for the current patients in Table II. The "same matches" column includes the number of patients who have been selected by both human clinicians and the automated agent. The "new matches" column gives the number of patients who have been matched
TABLE I

Results of Matching 187 Past patients.

\begin{tabular}{|c|c|c|c|}
\hline $\begin{array}{c}\text { Clinical } \\
\text { Trial }\end{array}$ & $\begin{array}{c}\text { Same } \\
\text { Matches }\end{array}$ & $\begin{array}{c}\text { New } \\
\text { Matches }\end{array}$ & $\begin{array}{c}\text { Missing } \\
\text { Data }\end{array}$ \\
\hline 10822 & 10 & 5 & 0 \\
10840 & 0 & 19 & 3 \\
11072 & 48 & 26 & 19 \\
11378 & 4 & 19 & 3 \\
11992 & 5 & 6 & 0 \\
12100 & 8 & 20 & 13 \\
12101 & 20 & 30 & 0 \\
\hline
\end{tabular}

TABLE II

RESUltS OF MATCHING 57 CURRENT PATIENTS.

\begin{tabular}{|c|c|c|c|}
\hline $\begin{array}{c}\text { Clinical } \\
\text { Trial }\end{array}$ & $\begin{array}{c}\text { Same } \\
\text { Matches }\end{array}$ & $\begin{array}{c}\text { New } \\
\text { Matches }\end{array}$ & $\begin{array}{c}\text { Missing } \\
\text { Data }\end{array}$ \\
\hline 11132 & 4 & 1 & 1 \\
11971 & 3 & 0 & 0 \\
12100 & 0 & 2 & 0 \\
12101 & 4 & 21 & 0 \\
12601 & 0 & 1 & 0 \\
11931 & 1 & 8 & 0 \\
12775 & 1 & 4 & 0 \\
\hline
\end{tabular}

by the agent but potentially missed by human clinicians. Finally, the last column shows the number of patients whose available records are incomplete. Clinicians have found trials for these patients, but the agent cannot identify these matches because of missing data. The agent has found a number of matches potentially missed by human clinicians; thus, it can help to recruit more patients for clinical trials.

In Table III, we give the mean test costs with and without the ordering heuristics for the 187 past patients. The results show that the implemented heuristics reduce the costs by more than a factor of two.

\section{SCALABILITy}

The time complexity of evaluating the acceptance and rejection expressions is linear in their size. Experiments on a Sun Ultra 10 have shown that the evaluation takes about 0.02 seconds per question, and the time is linear in the number of questions. Typical eligibility conditions for a clinical trial include ten to thirty questions; thus, the evaluation time is 0.2 to 0.6 seconds per trial.

TABLE III

COST SAVINGS BY TEST REORDERING.

\begin{tabular}{|c|c|c|}
\hline \multirow{2}{*}{$\begin{array}{c}\text { Clinical } \\
\text { Trial }\end{array}$} & \multicolumn{2}{|c|}{ Average Dollar Cost } \\
\cline { 2 - 3 } & Reordering & $\begin{array}{c}\text { With Test } \\
\text { Reordering }\end{array}$ \\
\hline 10822 & $\$ 20$ & $\$ 8$ \\
10840 & $\$ 0$ & $\$ 0$ \\
11072 & $\$ 556$ & $\$ 194$ \\
11378 & $\$ 34$ & $\$ 0$ \\
11992 & $\$ 87$ & $\$ 34$ \\
12100 & $\$ 0$ & $\$ 0$ \\
12101 & $\$ 24$ & $\$ 22$ \\
\hline
\end{tabular}




\begin{tabular}{|c|c|c|c|}
\hline $\begin{array}{l}\text { Adding patients } \\
\text { - Add a new patient } \\
\text { - Find an old patient }\end{array}$ & $\begin{array}{l}\text { Selecting dinical trials } \\
\text { - Choose candidate trials } \\
\text { - View available trials }\end{array}$ & $\begin{array}{l}\text { Entering initial data } \\
\text { - Answer initial questions } \\
\text { - Change previous answers }\end{array}$ & $\begin{array}{l}\text { Entering medical data } \\
\text { - Enter test results } \\
\text { - View eligibility decisions }\end{array}$ \\
\hline & & & $\begin{array}{l}\text { Revising medical data } \\
\text { - View test results } \\
\text { - Change some results }\end{array}$ \\
\hline
\end{tabular}

Fig. 4. Entering a patient's data. The web-based interface for data entry consists of five screens. We show these screens by rectangles and the transitions between them by arrows.

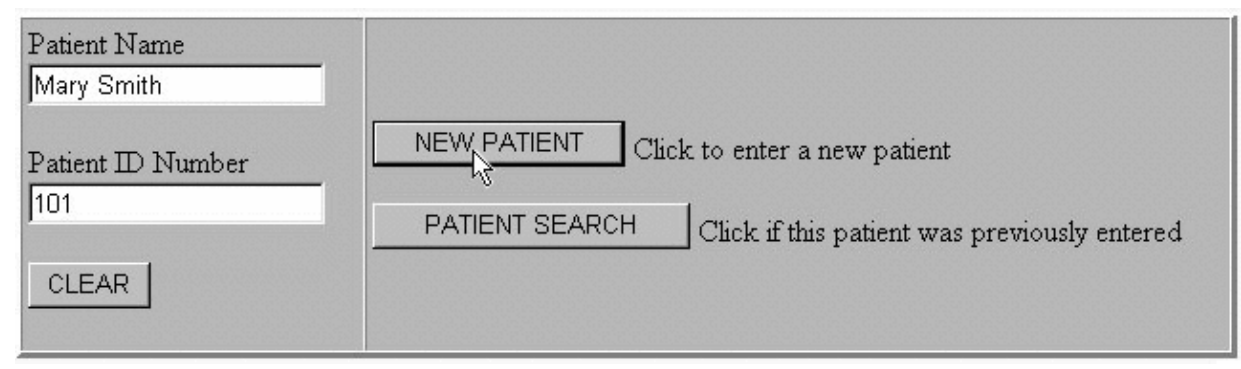

Fig. 5. Adding new patients and retrieving existing patients.

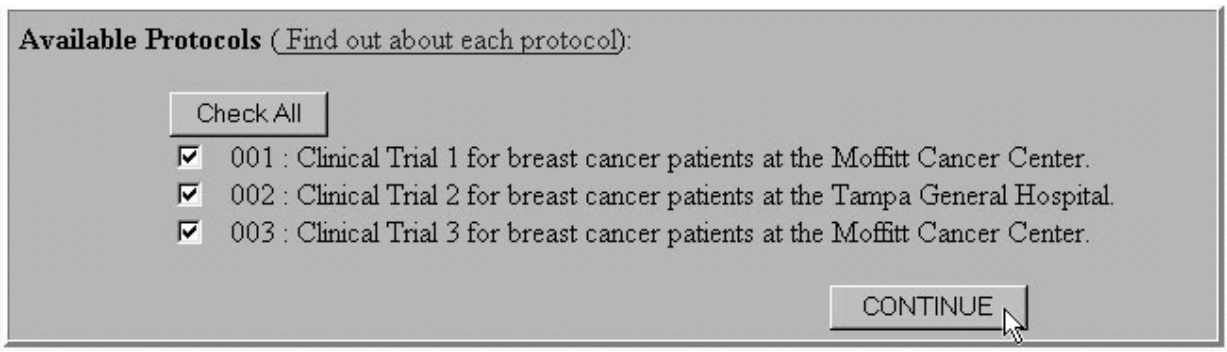

Fig. 6. Selecting clinical trials.

\begin{tabular}{|c|c|}
\hline What is the cancer stage? & What is the patients age? 40 \\
\hline How many nodes are positive? $\sqrt{2}$ & What is the greatest tumor diameter? 2.2 \\
\hline $\begin{array}{l}\text { Has the patient had surgery for breast cancer? } \\
C \text { Yes } \odot \text { No } \bigcirc \text { Defer }\end{array}$ & $\begin{array}{l}\text { Did the surgery include an axillary dissection? } \\
C \text { Yes } \odot \text { No } \odot \text { Defer }\end{array}$ \\
\hline What is the patients sex? Female & $\begin{array}{l}\text { Has the patient had any cancer therapy? } \\
\odot \text { Yes } \odot \text { No } \odot \text { Defer }\end{array}$ \\
\hline
\end{tabular}

Fig. 7. Entering basic information for a patient.

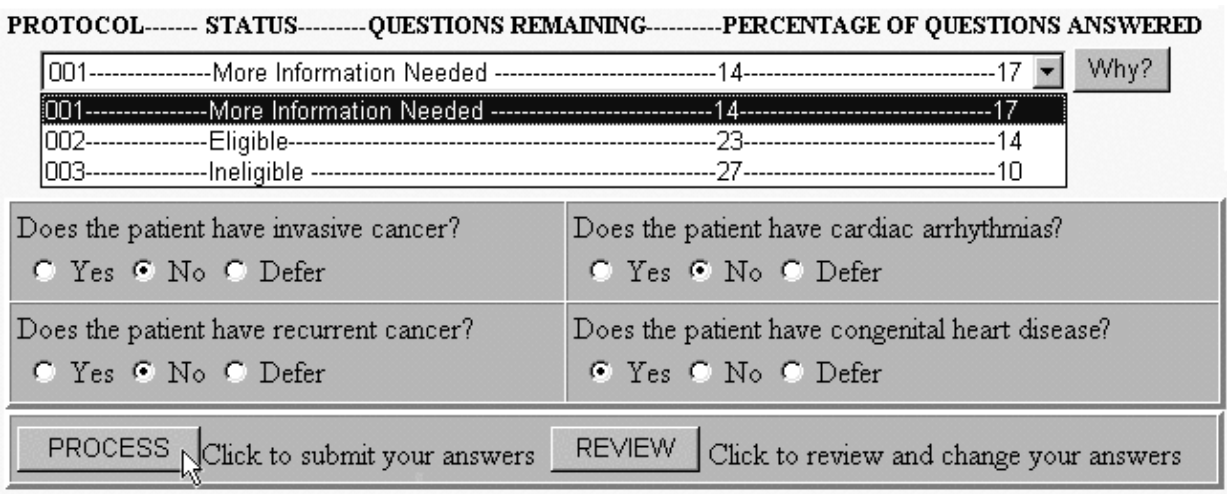

Fig. 8. Entering medical data. 


\section{(a) Eligibility criteria}

1. The patient is female.

2. She is at most forty-five years old.

3. Either

- her cancer is not invasive, or

- her cancer is not recurrent.

4. Either

- at most three lymph nodes have tumor cells, or

- all tumors are smaller than 2.5 centimeters.

5. Either

- the patient has no cardiac arrhythmias, or

- the patient has no congenital heart disease.

\section{(b) Acceptance expression}

sex $=$ FEMALE and

age $\leq 45$ and

( invasive $=\mathrm{NO}$ or recurrent $=\mathrm{NO})$ and

(lymph-nodes $\leq 3$ or tumor-size $\leq 2.5$ ) and

$($ arrhythmias $=\mathrm{NO}$ or congenital $=\mathrm{NO})$

\section{(c) Reduced expression}

sex $=$ FEMALE and

age $\leq 45$ and

invasive-and-recurrent $=$ NO and

(lymph-nodes $\leq 3$ or tumor-size $\leq 2.5$ ) and

arrhythmias-and-congenital $=\mathrm{NO}$

Fig. 9. Reducing the number of disjunctions. The conversion of the eligibility criteria (a) into a logical expression (b) leads to an explosion in the size of the corresponding disjunctive normal form. We can prevent the explosion by replacing some disjunctions with single questions (c).

The linear scalability is an important advantage over Bayesian systems, which do not scale to a large number of clinical trials [7, 21, 23]. The authors of these systems have reported that the sizes of the underlying networks are superlinear in the number of trials [22, 37], and the training time is superlinear in the network size [24, 34].

If the agent uses the cost-reduction heuristics, it converts the acceptance and rejection expressions into disjunctive normal form, which can potentially lead to an explosion in their size. For example, if eligibility conditions are as shown in Figure 9(a), the agent initially generates the expression in Figure 9(b). If the agent converts it to disjunctive normal form, the resulting expression consists of eight clauses.

Although the conversion may result in impractically large expressions, experiments have shown that this problem does not arise in practice because the number of nested disjunctions is usually small. Furthermore, we can eliminate some disjunctions by combining their elements into longer questions. For instance, we can represent Condition 3 in Figure $9(\mathrm{a})$ by a single question: "Does the patient have both invasive and recurrent cancer?" If we apply this modification to Conditions 3 and 5, then we obtain the expression in Figure 9(c), and its conversion to disjunctive normal form results in an expression with two clauses.

\section{Concluding Remarks}

We have developed an agent that automatically assigns patients to clinical trials. We have described the representation of selection criteria, heuristics for ordering of tests, and a web-based interface for entering patients' data, which will enable physicians across the country to access a central repository of clinical trials.

Experiments have confirmed that the agent has the potential to find more participants for clinical trials. They have also shown that the ordering of medical tests affects their overall cost, and the implemented heuristics can reduce the cost of finding trial participants. The heuristics do not account for the probabilities of possible test results, and we plan to add probabilistic reasoning as part of the future work.

Acknowledgments: This work has been partially supported by the Breast Cancer Research Program of the U.S. Army Medical Research and Materiel Command under contract DAMD17-00-1-0244, and by the H. Lee Moffitt Cancer Center.

\section{REFERENCES}

[1] D. Bareford and A. Hayling. Inappropriate use of laboratory services: Long term combined approach to modify request patterns. British Medical Journal, 301(6764):1305-1307, 1990.

[2] Sanjukta Bhanja, Lynn M. Fletcher, Lawrence O. Hall, Dmitry B. Goldgof, and Jeffrey P. Krischer. A qualitative expert system for clinical trial assignment. In Proceedings of the Eleventh International Florida Artificial Intelligence Research Society Conference, pages 84-88, 1998.

[3] Jacques Bouaud, Briggite Séroussi, Éric-Charles Antoine, Mary Gozy, David Khayat, and Jean-Franşois Boisvieux. Hypertextual navigation operationalizing generic clinical practice guidelines for patient-specific therapeutic decisions. Journal of the American Medical Informatics Association, 5(suppl.):488-492, 1998.

[4] Jacques Bouaud, Briggite Séroussi, Éric-Charles Antoine, Laurent Zelek, and Marc Spielmann. Reusing ONCODOC, a guideline-based decision support system, across institutions: A successful experiment in sharing medical knowledge. In Proceedings of the American Medical Informatics Association Annual Symposium, volume 7, 2000.

[5] Bruce G. Buchanan and Edward H. Shortliffe. RuleBased Expert Systems: The MYCIN Experiments of the Stanford Heuristic Programming Project. AddisonWesley, Reading, MS, 1984.

[6] Robert W. Carlson, Samson W. Tu, Nancy M. Lane, Tze L. Lai, Carol A. Kemper, Mark A. Musen, and Edward H. Shortliffe. Computer-based screening of patients with HIV/AIDS for clinical trial eligibility. Online Journal of Current Clinical Trials, 4(179), 1995.

[7] Francisco J. Díez, José Mira, E. Iturralde, and S. Zubillaga. DIAVAL, a Bayesian expert system for echocardiography. Artificial Intelligence in Medicine, 10(1):59-73, 1997.

[8] Lesley Fallowfield, D. Ratcliffe, and Robert Souhami. Clinicians' attitudes to clinical trials of cancer therapy. European Journal of Cancer, 33(13):2221-2229, 1997.

[9] John H. Gennari and Madhu Reddy. Participatory design and an eligibility screening tool. In Proceedings of the American Medical Informatics Association Annual Fall Symposium, pages 290-294, 2000.

[10] Carolyn Cook Gotay. Accrual to cancer clinical trials: 
Directions from the research literature. Social Science and Medicine, 33(5):569-577, 1991.

[11] Peter Hammond and Marek J. Sergot. Computer support for protocol-based treatment of cancer. Journal of Logic Programming, 26(2):93-111, 1996.

[12] M. Korver and A. R. Janssens. Development and validation of HEPAR, an expert system for the diagnosis of disorders of the liver and biliary tract. Medical Informatics, 16(3):259-270, 1993.

[13] M. Korver and Peter J. F. Lucas. Converting a rulebased expert system into a belief network. Medical Informatics, 18(3):219-241, 1993.

[14] Cyrus Kotwall, Leo J. Mahoney, Robert E. Myers, and Linda Decoste. Reasons for non-entry in randomized clinical trials for breast cancer: A single institutional study. Journal of Surgical Oncology, 50:125-129, 1992.

[15] Peter J. F. Lucas. Refinement of the HEPAR expert system: Tools and techniques. Journal of Artificial Intelligence in Medicine, 6(2):175-188, 1994

[16] Peter J. F. Lucas, R. W. Segaar, and A. R. Janssens. HEPAR: An expert system for the diagnosis of disorders of the liver and the biliary tract. Liver, 9:266-275, 1989.

[17] Michael D. McNeely and Beverly J. Smith. An interactive expert system for the ordering and interpretation of laboratory tests to enhance diagnosis and control utilization. Canadian Medical Informatics, 2(3):16-19, 1995.

[18] Ian R. Morrison, B. A. Schaefer, and Beverly J. Smith. Knowledge acquisition: The ACQUiRe approach. In Proceedings of the First Semi-Annual Conference in Policy Making and Knowledge Systems, 1991.

[19] Mark A. Musen. Automated Generation of Model-Based Knowledge Acquisition Tools. Morgan Kaufmann, San Mateo, CA, 1989.

[20] Mark A. Musen, Samson W. Tu, Amar K. Das, and Yuval Shahar. EON: A component-based approach to automation of protocol-directed therapy. Journal of the American Medical Informatics Association, 3(6):367$388,1996$.

[21] Lucila Ohno-Machado, Eduardo Parra, Suzanne B. Henry, Samson W. Tu, and Mark A. Musen. AIDS ${ }^{2}$ : A decision-support tool for decreasing physicians' uncertainty regarding patient eligibility for HIV treatment protocols. In Proceedings of the Seventeenth Annual Symposium on Computer Applications in Medical Care, pages 429-433, 1993.

[22] Agnieszka Oniśko, Marek J. Druzdzel, and Hanna Wasyluk. Learning Bayesian network parameters from small data sets: Application of noisy-Or gates. In Proceedings of the Workshop on Bayesian and Causal Networks: From Inference to Data Mining, 2000.

[23] Agnieszka Onisko, Mark J. Druzdzel, and Hanna Wasyluk. Application of Bayesian belief networks to diagnosis of liver disorders. In Proceedings of the Third Conference on Neural Networks and Their Applications, pages 730-736, 1997.

[24] Constantinos Papaconstantinou, Georgios Theocharous, and Sridhar Mahadevan. An expert system for assigning patients into clinical trials based on Bayesian networks. Journal of Medical Systems, 22(3):189-202, 1998.

[25] Franco Perraro, Paolo Rossi, Carlo Liva, Adolfo Bulfoni, G. Ganzini, and Adriano Giustinelli. Inappropriate emergency test ordering in a general hospital: Preliminary reports. Quality Assurance Health Care, 4:77-81, 1992.

[26] Briggite Séroussi, Jacques Bouaud, and Éric-Charles Antoine. Enhancing clinical practice guideline compliance by involving physicians in the decision process. In Werner Horn, Yuval Shahar, Greger Lindberg, Steen Andreassen, and Jeremy C. Wyatt, editors, Artificial
Intelligence in Medicine, pages 76-85. Springer-Verlag, Berlin, Germany, 1999.

[27] Briggite Séroussi, Jacques Bouaud, and Éric-Charles Antoine. Users' evaluation of ONCODOC, a breast cancer therapeutic guideline delivered at the point of care. Journal of the American Medical Informatics Association, 6(5):384-389, 1999.

[28] Briggite Séroussi, Jacques Bouaud, and Éric-Charles Antoine. ONCODOC: A successful experiment of computer-supported guideline development and implementation in the treatment of breast cancer. Artificial Intelligence in Medicine, 22(1):43-64, 2001.

[29] Briggite Séroussi, Jacques Bouaud, Éric-Charles Antoine, Laurent Zelek, and Marc Spielmann. Using ONCODOC as a computer-based eligibility screening system to improve accrual onto breast cancer clinical trials. In Silvana Quaglini, Pedro Barahona, and Steen Andreassen, editors, Artificial Intelligence in Medicine, pages 421-430. Springer-Verlag, Berlin, Germany, 2001.

[30] Edward H. Shortliffe. MYCIN: A Rule-Based Computer Program for Advising Physicians Regarding Antimicrobial Therapy Selection. PhD thesis, Computer Science Department, Stanford University, 1974.

[31] Edward H. Shortliffe, Randall Davis, Stanton G. Axline, Bruce G. Buchanan, Cordell C. Green, and Stanley Cohen. Computer-based consultations in clinical therapeutics: Explanation and rule acquisition capabilities of the MYCIN system. Computers and Biomedical Research, 8:303-320, 1975.

[32] Edward H. Shortliffe, A. Carlisle Scott, Miriam B. Bischoff, William van Melle, and Charlotte D. Jacobs. ONCOCIN: An expert system for oncology protocol management. In Proceedings of the Seventh International Joint Conference in Artificial Intelligence, pages 876$881,1981$.

[33] Beverly J. Smith and Michael D. McNeely. The influence of an expert system for test ordering and interpretation on laboratory investigations. Clinical Chemistry, 45(8):1168-1175, 1999

[34] Georgios Theocharous. An expert system for assigning patients into clinical trials based on Bayesian networks. Master's thesis, Computer Science and Engineering Department, University of South Florida, 1996.

35] Samson W. Tu, Carol A. Kemper, Nancy M. Lane, Robert W. Carlson, and Mark A. Musen. A methodology for determining patients' eligibility for clinical trials. Journal of Methods of Information in Medicine, 32(4):317-325, 1993.

[36] Carl Van Walraven and C. David Naylor. Do we know what inappropriate laboratory utilization is? A systematic review of laboratory clinical audits. Journal of the American Medical Association, 280(6):550-558, 1998.

[37] Haiqin Wang and Marek J. Druzdzel. User interface tools for navigation in conditional probability tables and elicitation of probabilities in bayesian networks. In Proceedings of the Sixteenth Conference on Uncertainty in Artificial Intelligence, pages 617-625, 2000.

[38] Salim Yusuf, Peter Held, K. K. Teo, and Elizabeth R. Toretsky. Selection of patients for randomized controlled trials: Implications of wide or narrow eligibility criteria. Statistics in Medicine, 9:73-86, 1990. 